\title{
THE CLASS 0 OUTFLOW HAMMERED OUT?
}

\author{
MICHAEL D. SMITH, ROLAND VÖLKER \\ GERHARD SUTTNER AND HAROLD W. YORKE \\ Astronomisches Institut der Universität Würzburg \\ Am Hubland, D-97074 Würzburg, Germany
}

\begin{abstract}
Dense molecular jets cutting through dense molecular clouds are simulated here using a ZEUS-type hydrodynamics code extended with molecular physics. $\mathrm{H}_{2}$ 'hot snapshots' and $\mathrm{CO}$ 'historical views' are nicely modelled with overdense uniform jets. Attention is drawn to some remaining hot problems: observed jet knots are bow shaped, bipolar outflows can be highly asymmetric, some proper motions within jets are enigmatically low and $\mathrm{H}_{2}$ excitation can be exceedingly uniform.

We present the Hammer Jet, in which the nozzle introduces high velocity variations, as well as a strong ripping and spray action. Prominent jet bow shocks in $\mathrm{H}_{2}$ and $\mathrm{CO}$ emission lines are then produced. Wide tubular $\mathrm{CO}$ structures with concave bases show up. Proper motions are simulated. The asymmetries are modelled by jet break-out from a molecular core into a diffuse atomic environment.
\end{abstract}

\section{Introduction}

The earliest outflows from protostars are well collimated and extremely long. Despite being deeply enshrouded by molecular gas, the warm shocked regions can be observed in the infrared $\mathrm{H}_{2}$ lines, thus revealing the present impact regions. The cool accumulated material can be observed in the submillimetre $\mathrm{CO}$ lines, delineating the total swept-up and swept-out material. The constraints imposed are encapsulated in Tables $1 \& 2$.

The hydrodynamic jet model for molecular flows has been developed and applied by Suttner et al. (1997) and Smith et al. (1997a). Its successes and failures are denoted by ticks and crosses in the boxes. The numerical hydrocode simulations include molecular dissociation and reformation. We found that three-dimensional models are necessary although one- and two- 
TABLE 1. Molecular Hydrogen signatures, typical examples and the successes (ticks) and failures (crosses) of the Suttner et al jet model

\begin{tabular}{|c|c|}
\hline $\begin{array}{c}\text { Multiple Bows } \\
\text { (L 1634, Davis et al 1997) }\end{array}$ & $\begin{array}{c}\text { Complex Filamentation } \\
\text { (HH 90/91, Davis et al 1994) } \\
\checkmark\end{array}$ \\
\hline $\begin{array}{l}\text { Twin/single-peaked profiles } \\
\text { (L 1448, Davis \& Smith 1996) } \\
\checkmark\end{array}$ & $\begin{array}{c}\text { Constant Excitation } \\
\text { (Cepheus E, Eislöffel et al 1996) } \\
\times\end{array}$ \\
\hline $\begin{array}{c}\text { Pulsed jets } \\
\text { (HH 111, Gredel \& Reipurth 1993) } \\
\qquad / X\end{array}$ & $\begin{array}{c}\text { Asymmetric Lobes } \\
\text { (L 1660, Davis et al 1997) } \\
\checkmark / x\end{array}$ \\
\hline
\end{tabular}

dimensional models can provide some insight. The high number of ticks is encouraging.

One major problem with our early simulations is the structure and number of the jet knots. As shown in the pulsed jet of Fig. 2, the model knots are few, weak and elliptical. Although this is not inconsistent with all the observations, the HH 111 jet possesses many knots and the $\mathrm{HH} 212$ jet has inter-knot emission. Moreover, knots are usually bow shaped (Reipurth et al 1997, and these proceedings).

\section{The Hammer Jet}

Nozzle designs which we have recently studied are cartooned in Fig. 1. The parameters for all four models discussed here are given in Table 3. The new models include high-amplitude pulsations and are thus termed 'Hammer Jets'. They also include a $50 \%$ velocity shear, with the velocity in the jet sheath being lower than on the axis (as might be expected from disk-wind models). The result is: prominent outward-facing bow shocks, and extended wakes (Fig. 2). Also found are (1) wider outflows and stronger $\mathrm{H}_{2}$ emission (due to the chosen spray and precession angles), (2) more structure in the leading bow shock, including mini-bows, (3) wave patterns on the cavity surface and (4) the highest surface brightness knots in the lobe fall well behind the leading edge.

The bows are all concave to the jet origin. In contrast, if the largest 
TABLE 2. CO signatures and the successes (ticks) and failures (crosses) of the Smith et al jet model. NGC 6334G (Lada \& Fich 1996) displays all these signatures.

\begin{tabular}{||c|c}
\hline Collimation increases with Velocity & Red/blue lobe symmetry (high-v) \\
\hline \begin{tabular}{c|c} 
Power-law line profiles $(\gamma=-1.3$ to -2.0$)$ \\
$\checkmark$
\end{tabular} & Hubble Law $(v \propto$ distance) \\
\hline High-speed CO Jets \& Bullets & Strong Forward Motion \\
$\checkmark$ & $\checkmark$
\end{tabular}

velocity variations are applied to the inner axial region while the jet sheath maintains smaller pulsations, concave and convex bow shocks appear. Figure 3 demonstrates this aptly, where knot collisions (boxes d-h) generate intricate structures. Jet production models can in principle be constrained. For example, de Laval nozzles should possess lower axial speeds corresponding to the higher axial pressure. Clear observed examples of reverse bow shocks, however, have not been found although HH 11 and HH 2 could be cited.

TABLE 3. Jet parameters for the models displayed. The average jet axis speed is $100 \mathrm{~km} / \mathrm{s}$, jet density is $10^{5} \mathrm{~cm}^{-3}$, ambient density is $10^{4} \mathrm{~cm}^{-3}$, both initially fully molecular.

\begin{tabular}{|l|c|c|c|c|}
\hline \multirow{2}{*}{ Model Label } & $\begin{array}{c}\text { Shear } \\
\mathbf{v}_{o} / \mathbf{v}_{i}\end{array}$ & $\begin{array}{c}\text { Spray } \\
\psi\end{array}$ & $\begin{array}{r}\text { Precession } \\
\mathrm{P}(\mathrm{yrs}), \beta\end{array}$ & $\begin{array}{c}\text { Pulsation } \\
\mathrm{Q}(\mathrm{yrs}), \mathrm{A}(\mathrm{km} / \mathrm{s})\end{array}$ \\
\hline 3D-1 & - & - & $100,1^{\circ}$ & - \\
3D-2 & - & - & $100,1^{\circ}$ & 50,30 \\
MODEL 1 & $50 \%$ & - & $46,1^{\circ}$ & 50,90 \\
MODEL 2 & $50 \%$ & $2^{\circ}$ & $36,2^{\circ}$ & 40,90 \\
\hline
\end{tabular}



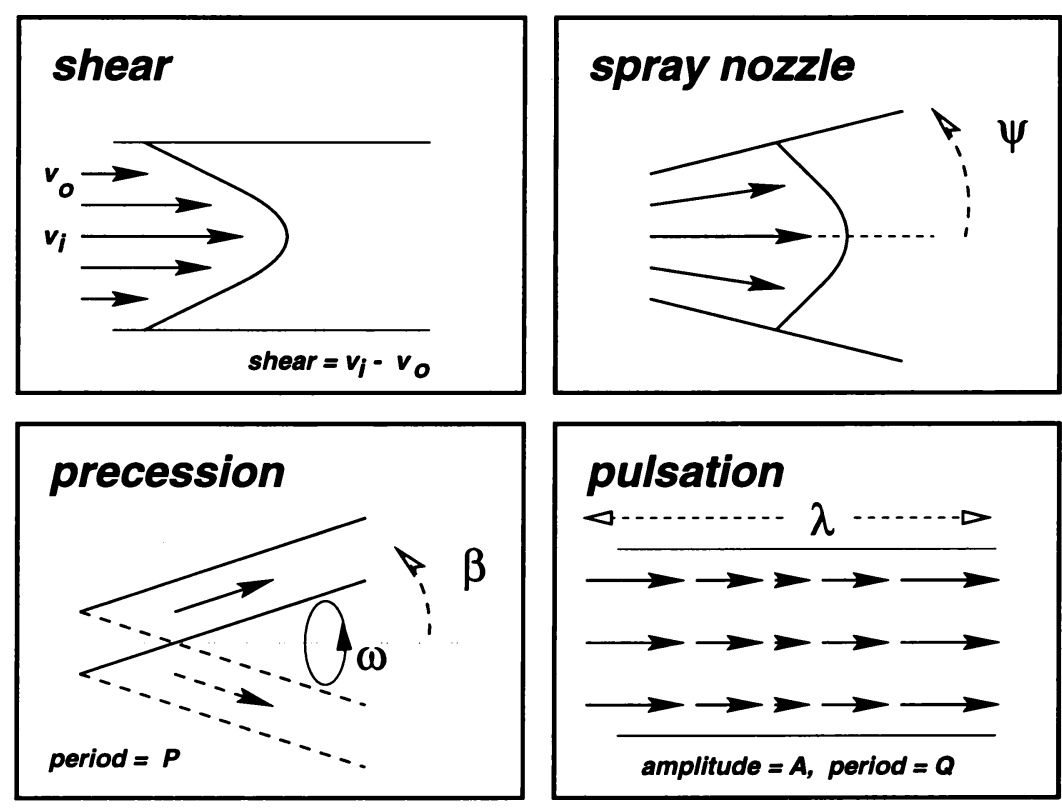

Figure 1. Nozzle geometry for the simulations

\section{Lobe Asymmetries}

$\mathrm{H}_{2}$ asymmetry is not directly predicted by our one-sided models. Nevertheless, asymmetries can be incorporated either through jet-related asymmetry or environmental asymmetry.

First, we study jet asymmetry. In Fig. 4 we present MODEL 2, as it would appear in the two perpendicular planes. Also note that the axis is aligned to $60^{\circ}$ out of the sky plane. Fine-scale asymmetries are produced. Prominent are the wave structure and sheath asymmetries, comparable to some observations (e.g. HH 211, McCaughrean et al 1994).

Large-scale asymmetries are clearly a mark of the environment. We can suppose that one jet approaches the cloud edge, and undergoes the transition from molecular cloud to atomic diffuse inter-cloud gas, as schematically shown in Figure 5. One then only sees the jet shock in $\mathrm{H}_{2}$. The result of our 2D simulation is shown in Fig 6. The jet survives the transition but is broadened. However, 3D simulations are required before generation of $\mathrm{H}_{2}$ distributions would be fruitful. 
$\sim$ (5 $^{601-}$

$\simeq$ 으 $\infty$
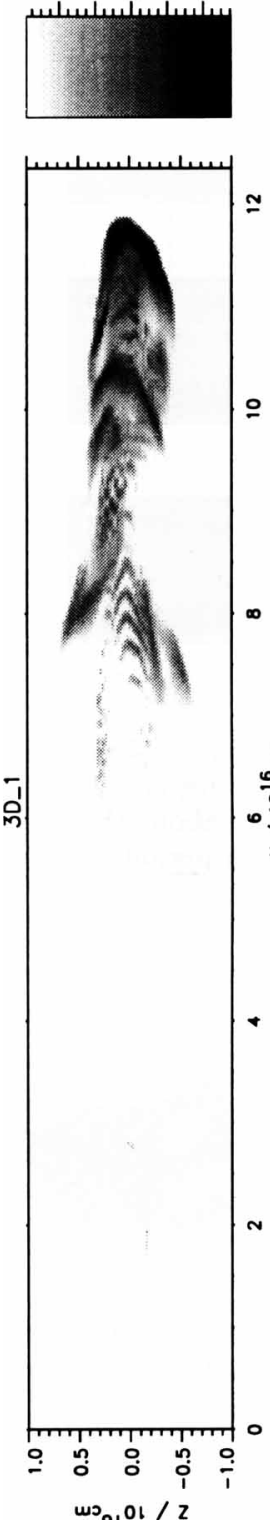

س० $01 / \mathrm{Z}$

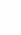

(6) $601-$

$\simeq$ 우 $0+$
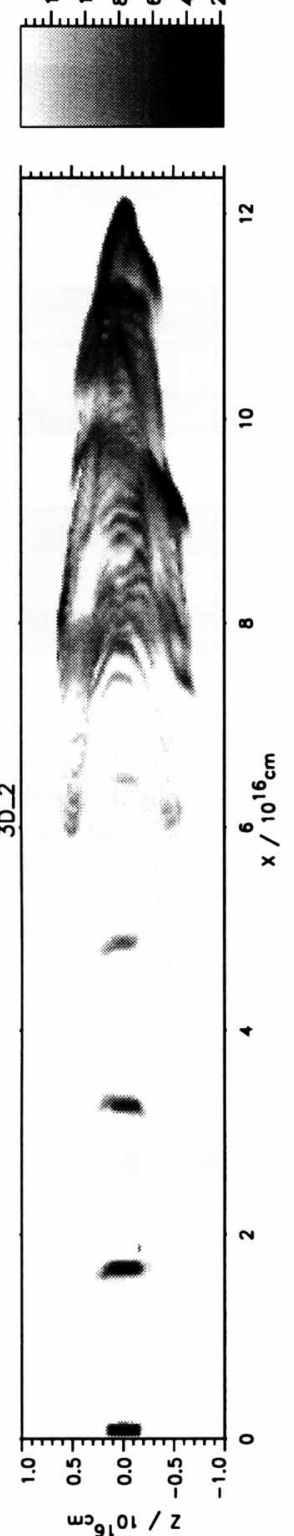

wo 91 l / Z
(6) $601-$
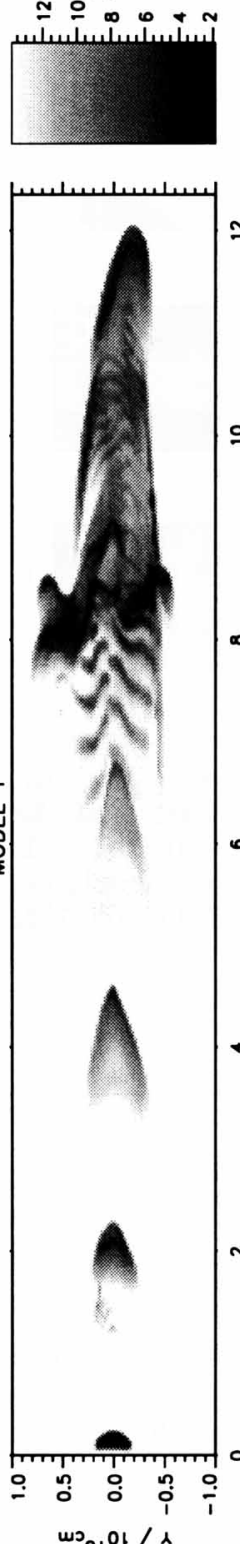

0
0
0
0
0
0
$\times 0$

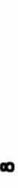

0
0
0
0
0
0
$\times 0$

늠

(s) $601-$

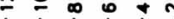
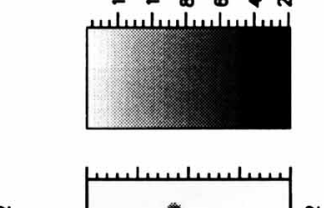

N

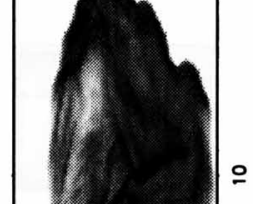

f.

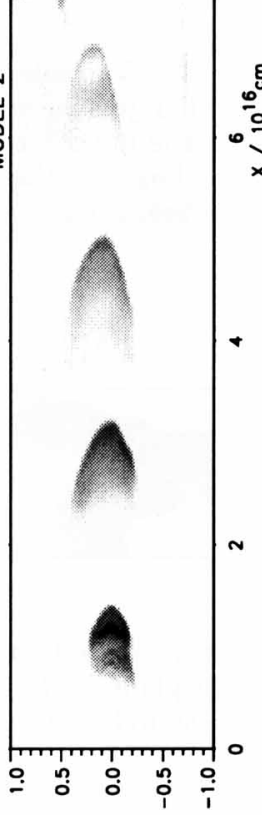

wo $01 / 1$

Figure 2. The four jet models at a comparable size in molecular hydrogen emission (from Suttner et al (1997) \& Völker et al (1997)). The Cartesian grid is $500 \times 80 \times 80$ zones, the jet radius is $1.5 \times 10^{15} \mathrm{~cm}$ or 6 zones. 

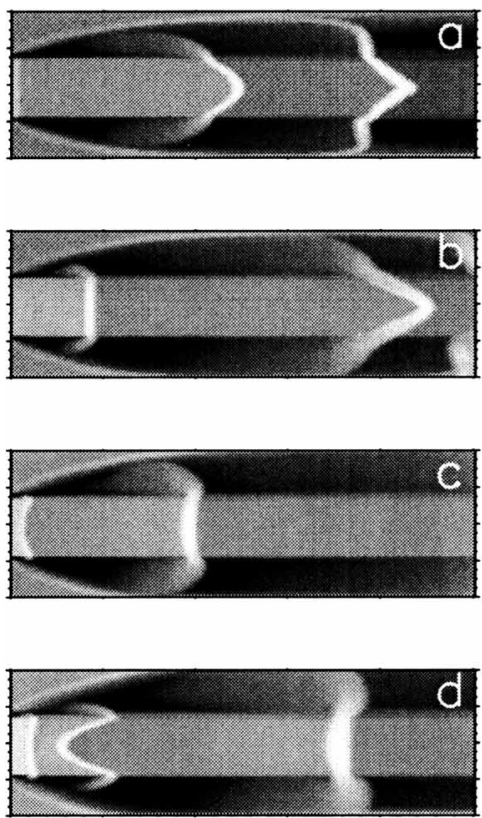
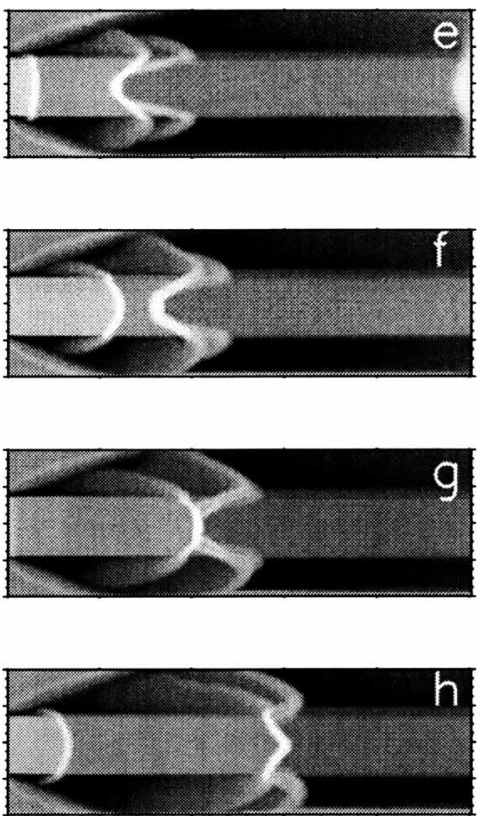

Figure 3. Variable Shear Jets. The density distributions for a sequence of 2D (Cartesian) molecular jets in which the cross-jet shear is given both positive and negative values. While the outer jet layer is injected with $90 \% 25 \mathrm{yr}$ periodic variations about the speed of $100 \mathrm{~km} \mathrm{~s}^{-1}$, the inner jet modulates this by a further $75 \%$ with a period of $61 \mathrm{yr}$. Times are, from (a) to (h), 149, 166, 183, 208, 230, 240, 248 \& $258 \mathrm{yr}$.

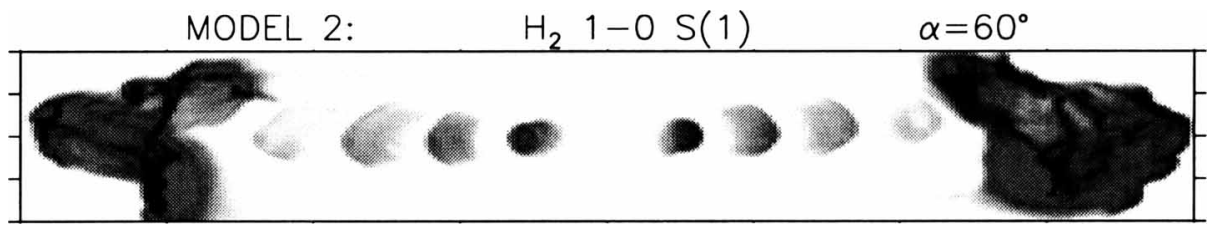

Figure 4. The twin-jet: MODEL 2 seen at $60^{\circ}$ to the sky plane and in the two perpendicular planes (left: XZ, right: XY). See Völker et al (1997)). Note the round non-aligned bows, as well as the lobe asymmetry.

\section{Tubular CO}

One complaint about the jet model has been that it cannot produce wide CO structures since strong molecular cooling would shrink the cocoon. This is not true: an extremely low density and very broad atomic gas cocoon 


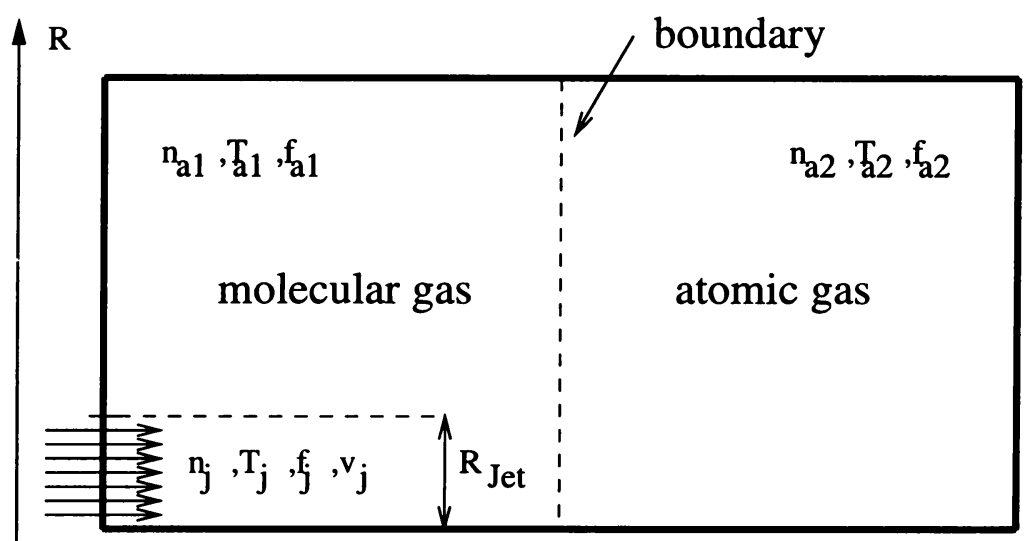

$\mathrm{Z}$

Figure 5. The initial conditions for the axially-symmetric calculation of molecular jet 'break-out' from a dense molecular core into an atomic ambient medium. Here $\mathrm{n}_{j}=\mathrm{n}_{a 1}$ $=10^{5} \mathrm{~cm}^{-3}$ and $\mathrm{n}_{a 2}=68.2 \mathrm{~cm}^{-3} \cdot \mathrm{T}_{a 1}=10 \mathrm{~K}, \mathrm{~T}_{a 2}=8000 \mathrm{~K}$.

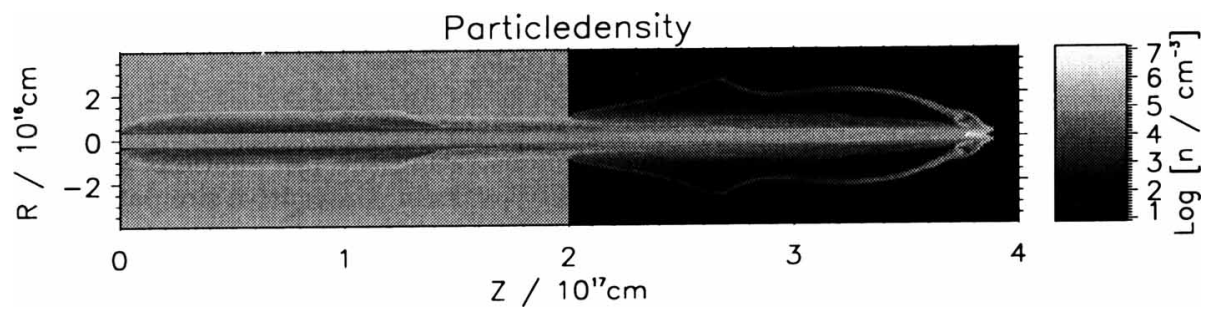

Figure 6. After 2000 years, the jet has broken well out of the core. A wide bow shock is present - note the increase in cocoon size at the boundary. The jet shock is extremely weak and the jet molecules are driven sideways into the atomic medium.

is trapped between the jet and the swept-up shell of cool gas. The jet is hypersonic $(\mathrm{M}=200)$ and the swept-up shell too inertial to squeeze out the cocoon. The result is a tubular CO structure many times wider than the jet (Fig. 7). Note also the concave structure at the jet base. We thus find good agreement with the observations of low-velocity gas (e.g. of HH 111, Nagar et al 1997) as well as the (usually very weak) high-velocity component (Smith et al 1997). CO lobe and jet predictions from zero (Suttner et al 1997) to high speeds (Smith et al 1997) are in remarkable agreement with observations. 


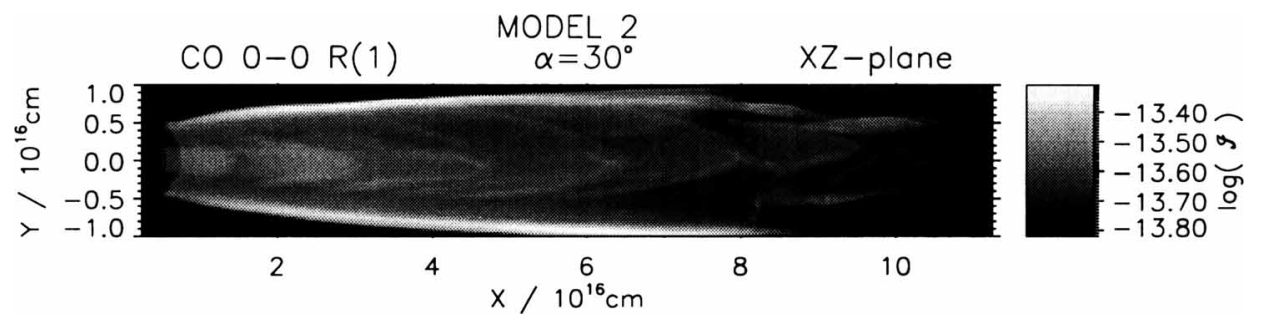

Figure 7. $\mathrm{CO}$ emission from the $\mathrm{J}=2-1$ transition for MODEL 2. The integrated $C O$ emission shown here is dominated by the low velocity tube, several times wider than the jet. Some $\mathrm{CO}$ bullets are also evident (the same $\mathrm{CO} / \mathrm{H}_{2}$ ratio in the jet and ambient gas is here assumed). Note that the high velocity $C O$ distributions appear very different (Smith et al 1997).

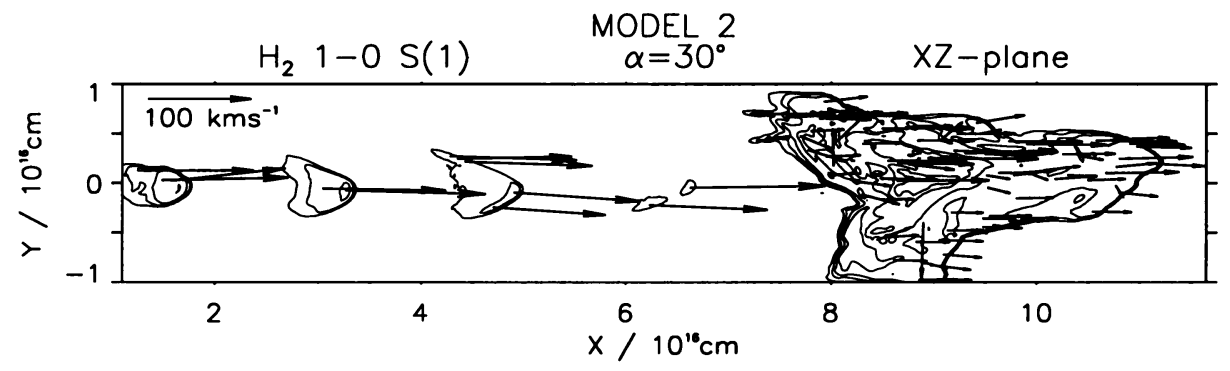

Figure 8. Proper motions in MODEL 2, demonstrating the fast motions within the jet. Proper motions were calculated from intensity peaks in consecutive simulated $\mathrm{H}_{2}$ 1-0 $\mathrm{S}(1)$ images.

\section{Proper Motions}

Individual knots in observed jets possess high proper motions. Data on the $\mathrm{H}_{2}$ jets are lacking, but in optical jets knot pattern speeds (i.e. from the proper motions) appear to be lower than predicted by the fluid speeds (from the radial speeds) (Eislöffel 1994, Eislöffel et al 1994). In the HH 34 jet, the ratio of the pattern speeds of the jet bows and their fluid speeds are not too discrepant $(0.7-0.9)$. The ratio in several jets increases with propagation distance from values as low as 0.2 towards unity. In contrast, models to date predict that the fluid and pattern speeds do not widely differ in the jet.

However, the hammer jet shown in Figures 8 and 9 possesses fluid speeds of up to $190 \mathrm{~km} \mathrm{~s}^{-1}$, yet proper motions for the jet bows are $110-150 \mathrm{~km} \mathrm{~s}^{-1}$. If one assumes that the (projected) knot fluid speed corresponds to the highest observed radial speed, then pattern-to-fluid speed ratios of 0.6-0.8 


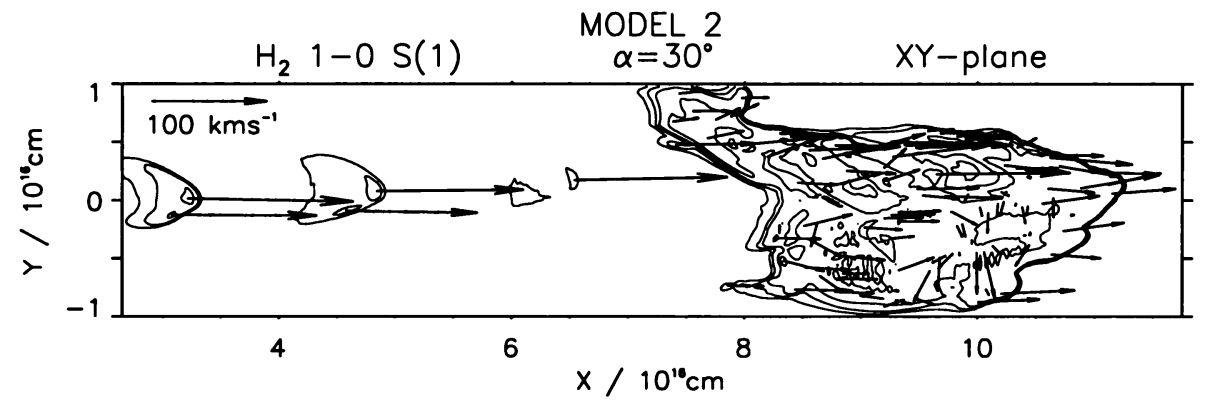

Figure 9. Proper motions in $\mathrm{H}_{2}$ for MODEL 2, observed from along the Z-axis, otherwise as described in Fig. 8,

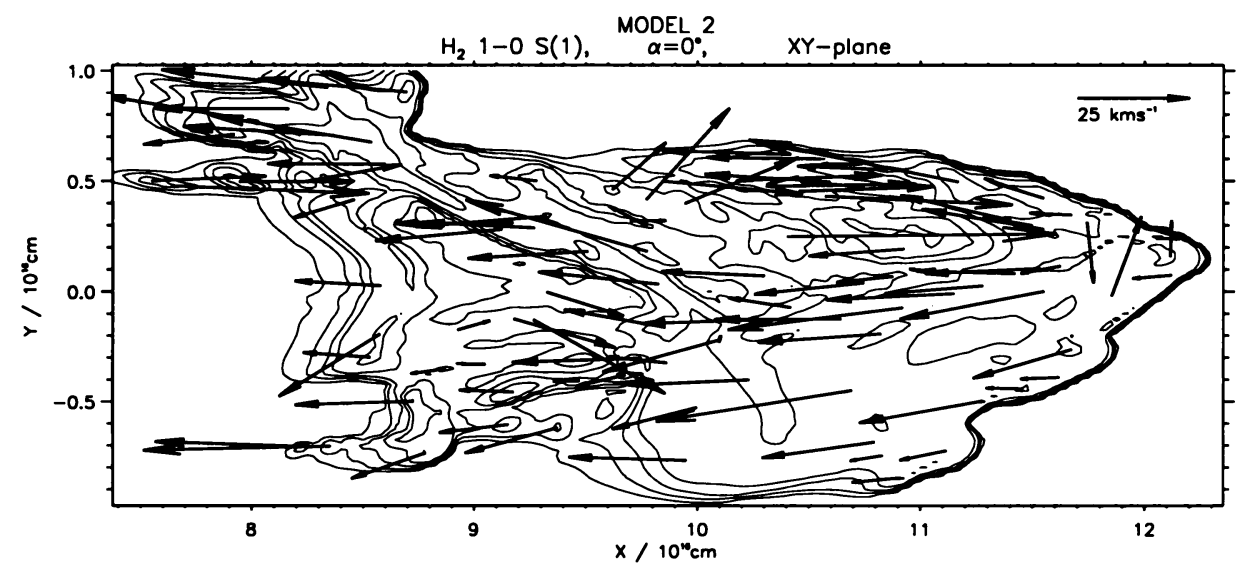

Figure 10. Proper motions in a magnified head region, demonstrating the flow away from the leading edge. That is, the average speed of the whole outflow has been subtracted. Proper motions were calculated from intensity peaks in consecutive simulated $\mathrm{H}_{2}$ 1-0 $\mathrm{S}(1)$ images.

would be predicted for this simulation. The high radial speeds are indeed present on the source-side of the two curved shocks (forward and reverse) which clash at each bow-shaped knot. Moreover, as the jet shocks weaken with propagation distance, pattern-to-fluid ratios should increase towards unity along the jet. We hope to confirm these predictions rigorously in the near future (Völker et al 1997) .

The proper-motion situation is more intricate in the lobes. Here the 
simulated molecular flow seen relative to the apex (Fig. 10) possesses both an acceleration away from the leading edge as well as a turbulent characteristic. These are the properties observed in the optical (Eislöffel et al 1994). HST and ground-based observations will soon provide us with the infrared data we need.

\section{Conclusions}

The various signatures of the collimated Class 0 outflows have here been simulated and discussed. Hammer Jets with shear provide new solutions to old problems. Remaining questions include:

- How and where do the jets form?

- How and where do the magnetic field, ambipolar diffusion and angular momentum change the picture presented above?

- Do the jets form complete with molecules? Or are the molecules entrained or formed on the jet dust?

- Can we explain the Gigantic Bipolar Outflows?

- How do outflows evolve over the Class 0 and Class I lifetimes?

- Can these outflows alter the environment sufficiently to influence the further formation of stars?

Further simulations of molecular hammers may provide some answers.

Acknowledgements: The authors gratefully acknowledge the support of the DFG.

\section{References}

Davis,C.J., Mundt, R., Eislöffel, 1994, ApJL, 437, L55.

Davis,C.J., Ray, T.P, Eislöffel, J., Corcoran, D., A\&A 1997, in press.

Davis, C.J., Smith, M.D., 1996, A\&A, 309,929.

Eislöffel, J., 1994, Ap\&SS, 216, 135.

Eislöffel, J., Mundt, R., Böhm, K-H., 1994, AJ, 108,1042.

Eislöffel, J., Smith, M.D., Davis, C.J., Ray, T.P., 1996, AJ, 112, 2086.

Gredel, R., Reipurth, B., 1993, ApJ, 407, L29.

Lada, C.J., Fich, M., 1996, ApJ, 459, 638.

McCaughrean, M.J., Rayner, J.T., Zinnecker, H., 1994, ApJ, 436, L189.

Nagar, N.M., Vogel, S.N., Stone, J.M. \& Ostriker, E. 1997, preprint (see also IAU Symp. 182, Poster Book, p 163).

Reipurth, B., Hartigan, P., Heathcote, S., Morse, J.A. \& Bally, J., 1997, AJ, in press

Smith,M.D., Suttner, G., Zinnecker, H., 1997, A\&A, in press.

Suttner, G., Smith, M.D., Yorke, H.W., Zinnecker, H., 1997, A\&A, 318, 595 